\title{
Short interfering RNA-mediated silencing of actin-related protein $2 / 3$ complex subunit 4 inhibits the migration of SW620 human colorectal cancer cells
}

\author{
XIAOJUAN SU*, SIYANG WANG*, YONGXU HUO and CHUNLEI YANG \\ College of Life Sciences, Medical Biology Department, Sichuan University, Chengdu, Sichuan 610041, P.R. China
}

Received January 26, 2016; Accepted October 26, 2017

DOI: $10.3892 / \mathrm{ol} .2017 .7642$

\begin{abstract}
Actin-related protein 2/3 complex subunit 4 (ARPC4) acts as an actin nucleator in actin cytoskeleton branching and contributes to cell migration. ARPC4 has previously been demonstrated to be abnormally expressed in various colorectal carcinoma cell lines, particularly SW620 cells. The present study explored the biological action and the possible mechanisms underlying the function of ARPC4 in the progression of carcinoma. The proliferation and migration of SW620 cells transfected with ARPC4-specific short interfering (si)RNAs were assessed using western blot, cell counting, flow cytometry and transwell assays. SW620 cells exhibited the highest ARPC4 expression of the cell lines investigated, and siRNA538 was the most effective of the siRNAs considered. The results of the present study demonstrated that ARPC4-silencing exhibited a significant effect on the capacity of cells for migration, but did not affect their proliferative ability. ARPC4-silencing inhibited human SW620 cell migration, but not proliferation, in vitro, suggesting that ARPC4 may be a putative therapeutic target for colorectal carcinoma.
\end{abstract}

\section{Introduction}

Colorectal cancer is a major cause for morbidity and mortality and one of the most common malignant tumours of the digestive system ( $\sim 9.7 \%$ of cases), thus posing a serious threat to human physical and mental health (1). The most hazardous characteristics of colorectal cancer are invasion and metastasis. Although basic and clinical cancer research has made notable progress in recent years, the survival rate for the majority of patients with cancer has not significantly improved, with

Correspondence to: Professor Chunlei Yang, College of Life Sciences, Medical Biology Department, Sichuan University, 17 Renmin South Road, Chengdu, Sichuan 610041, P.R. China

E-mail: yangchunlei@scu.edu.cn

${ }^{*}$ Contributed equally

Key words: actin-related protein 2/3 complex subunit 4, colorectal carcinoma, migration, proliferation metastasis and relapse following treatment being the cause for $>90 \%$ of cancer-associated mortality (2). The current clinical treatment for tumour metastasis exhibits numerous adverse effects, and there is a lack of systematic and comprehensive knowledge regarding the biological mechanisms underlying tumour metastasis. Therefore, prediction, early diagnosis and effective interventions to prevent tumour metastasis to distal tissues or organs are limited. In order to improve the effects of therapeutics on malignant tumours, the molecular mechanisms underlying tumour invasion and metastasis should be investigated (3-5).

Previously, research into metastasis focused on the actin cytoskeleton and increases in actin-related protein (ARP) expression during metastasis. The ARP2/3 complex is an actin-assembly nucleating agent that promotes the nucleation of microfilaments and serves a significant function in a number of physiological activities, including cell migration (6,7). Cell migration is essential to normal biological processes, such as tissue repair and regeneration; however, abnormally activated cell movement is associated with various diseases and may eventually lead to the development of fatal metastatic tumours. Indeed, metastatic capacity is considered a cancer cell marker (8-10).

A previous study demonstrated that ARP subunits are abnormally expressed in tumours, and immunohistochemical experiments indicated that abnormally increased levels of ARPC4 were present in colorectal cancer tissues (1). Investigating the association between ARPC4 and tumours may, therefore, provide valuable insights for the development of strategies for early cancer diagnosis and gene therapy. Molecular and cell biology studies previously demonstrated abnormally increased ARPC4 expression levels in various colorectal cancer cell lines; however, the association between the ARPC4 gene and the occurrence and development of colorectal cancer has not yet been fully elucidated. In the present study, further explorative research was conducted to identify novel targets for colorectal cancer gene therapy.

\section{Materials and methods}

Tissue samples. Colorectal carcinoma and adjacent normal colon tissue were obtained from a female patient at the age of 67 in August 2015 by resection at The Third People's Hospital of Chengdu (Chengdu, China). Written informed consent was 
obtained from the patient and ethical approval was granted by the Medical Ethics Committee of The Third People's Hospital of Chengdu. Immunohistochemical staining images were analysed with Imaris 8.01 (Bitplane AG, Zurich, Switzerland).

Immunohistochemical analysis. Detection of ARPC4 protein levels was performed as follows: A paraffin section of the tumour sample 6-8- $\mu$ m thick was dewaxed with xylene I and II for $5 \mathrm{~min}$ at room temperature, then incubated with descending ethanol series $(100,95,90,80$ and 70\%) for 3-5 min. Slices were rinsed twice with distilled water and then use PBS to rinse two to three times with PBS. $\mathrm{H}_{2} \mathrm{O}_{2}$ solution (3\%) was used to block the endogenous peroxidase activity for $10 \mathrm{~min}$ at room temperature, followed by high-pressure antigen repair. It was sealed using 10\% goat serum (cat no. ab7481; Abcam, Cambridge, UK) and the primary monoclonal rabbit anti-ARPC4 antibody (dilution, 1:100; cat no. ab217065; Abcam) was added, then incubated at $4^{\circ} \mathrm{C}$ overnight and washed three times with PBS. The biotin-labelled mouse anti-rabbit IgG secondary antibody (dilution, 1:100; cat no. ab6728; Abcam) was added and incubated for $30 \mathrm{~min}$ at room temperature, then washed three times

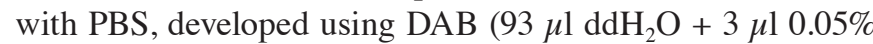
DAB1 solution $+2 \mu 10.05 \%$ DAB2 solution $+2 \mu 10.05 \%$ DAB3 solution) and re-dyed using $2 \%$ haematoxylin at $4{ }^{\circ} \mathrm{C}$ for $10 \mathrm{~min}$. It was then sealed by neutral gum and imaged under a microscope (BX-42; Olympus Corporation, Tokyo, Japan).

Using light microscopy at magnification, x400, cells from 3 fields of view were analysed and the average number of positive cells was noted. According to the following scoring standard, the number of positive stained cells was calculated and divided into the ARPC4 low-expression group ( $<10 \%$ positive cells) or the ARPC4 high-expression group ( $>10 \%$ positive cells). This was calculated by the following formula: Number of positively stained cells/total number of cells x 100 .

Cell culture conditions and reagents. The human colorectal cancer SW620, HT-29, HCT116 and SW480 cell lines were obtained from the Institute of Biochemistry and Cell Biology (Shanghai, China) and maintained by the laboratory within the Medical Biology Department, Sichuan University (Chengdu, China). SW620, HT-29, HCT116 and SW480 cell lines were cultured with high-glucose Dulbecco's modified Eagle's medium (DMEM; HyClone; GE Healthcare Life Sciences, Logan, UT, USA ) containing 10\% fetal bovine serum (FBS; Gibco; Thermo Fisher Scientific, Inc., Waltham, MA, USA) and penicillin-streptomycin (10,000 U/ml; 1:100; Gibco; Thermo Fisher Scientific, Inc.) in a Heracell ${ }^{\mathrm{TM}}$ VIOS 160i $\mathrm{CO}_{2}$ incubator (Thermo Fisher Scientific, Inc.), and were maintained in a $5 \% \mathrm{CO}_{2}$ atmosphere at $37^{\circ} \mathrm{C}$. all the cells were replaced with new medium every 2 days and passaged when the confluence reached $\sim 90 \%$ by using $0.25 \%$ trypsin (Gibco; Thermo Fisher Scientific, Inc.).

Cell culture conditions and reagents. SW620 cells were maintained at $37^{\circ} \mathrm{C}$ in an atmosphere containing $5 \% \mathrm{CO}_{2}$ in Dulbecco's modified Eagle's medium (DMEM; HyClone; GE Healthcare Life Sciences, Logan, UT, USA) supplemented with $10 \%$ foetal bovine serum (FBS; Gibco; Thermo Fisher Scientific, Inc., Waltham, MA, USA). Lipofectamine ${ }^{\circledR} 2000$ was purchased from Life Sciences (Thermo Fisher Scientific,
Inc.). Primary antibodies against $\beta$-actin (cat no. ab8227) and ARPC4 were purchased from Abcam (Cambridge, UK). Primary antibodies for vimentin (cat. no. 10366-1-AP), E-cadherin (cat. no. 20874-1-AP) and PCNA (cat. no. 24,036-1-AP) were supplied by ProteinTech Group, Inc. (Chicago, IL, USA). Secondary antibodies against vimentin (cat.no. sc-2370), E-cadherin (cat. no. sc-2030) and PCNA (cat. no. sc-2995) were purchased from Santa Cruz Biotechnology Inc. (Dallas, TX, USA).

Western blot assay. HT-29, HCT-116, SW480, SW620, SW-1116 or transfected and null SW620 colorectal cancer cells were harvested $48 \mathrm{~h}$ following transfection, during the logarithmic growth phase, and total cellular protein was extracted. All cells were washed three times with PBS, centrifuged at $1,000 \mathrm{x} g$ for $5 \mathrm{~min}$ at room temperature, and re-suspended in radioimmunoprecipitation assay lysis (Beijing Solarbio Science \& Technology, Beijing China) and extraction buffer containing $1 \mathrm{mM}$ phenylmethylsulfonyl fluoride protease inhibitor (Beijing Solarbio Science \& Technology) on ice for $30 \mathrm{~min}$ to ensure completely lysis. A total volume of $5 \mathrm{ml} 5 \mathrm{X}$ loading buffer [(250 mM Tris-HCL (pH 6.8), 10\% (w/v) SDS, 0.5\% (w/v) BPB, $50 \%(\mathrm{v} / \mathrm{v})$ glycerin and $5 \%(\mathrm{w} / \mathrm{v}) \beta$-mercaptoethanol)] was added and boiled at $100^{\circ} \mathrm{C}$ for $5-10 \mathrm{~min}$. then centrifuged at $12,000 \mathrm{x} \mathrm{g}$ for $15 \mathrm{~min}$ at $4^{\circ} \mathrm{C}$ to collect the supernatant. The total protein concentration was detected using the bicinchoninic acid method (BCA) (Beyotime Institute of Biotechnology, Haimen, China). A total of $40 \mu \mathrm{g}$ protein for each sample was uploaded and separated by SDS-PAGE, with a 5\% stacking gel and $12 \%$ separating gel, and transferred to polyvinylidene fluoride membranes (EMD Millipore, Billerica, MA, USA). The membranes were then blocked with TBS containing $0.05 \%$ Tween-20 (TBST) and $5 \%$ non-fat milk for $1 \mathrm{~h}$ at room temperature. Polyclonal rabbit anti- $\beta$ actin (dilution, 1:200), rabbit monoclonal anti-ARPC4 (dilution, 1:200), polyclonal rabbit anti-vimentin antibody (dilution, 1:200), rabbit polyclonal anti-E-cadherin antibody (dilution, 1:200) and PCNA rabbit polyclonal antibody (dilution, 1:200) antibodies were added to incubate at $4^{\circ} \mathrm{C}$ overnight. Following washing three times for $10 \mathrm{~min}$ each with TBST, the membranes were incubated with horseradish peroxidase (HRP)-conjugated goat anti-rabbit (dilution, 1:5,000; cat no. A8275; Sigma-Aldrich; Merck KGaA, Darmstadt, Germany) and peroxidase-conjugated goat anti-rabbit (dilution, 1:5,000; cat no. A0418; Sigma-Aldrich; Merck KGaA), goat anti-rabbit IgG-HRP secondary antibody (dilution, 1:5,000; cat no. sc-2370; Santa Cruz Biotechnology, Inc.), goat anti-rabbit IgG-HRP secondary antibody (dilution, 1:5,000; cat no. sc-2030; Santa Cruz Biotechnology, Inc.) and chicken anti-rabbit IgG-HRP secondary antibody (dilution, 1:5,000; cat no. sc-2955; Santa Cruz Biotechnology, Inc.) for $1 \mathrm{~h}$ at room temperature. Subsequent to washing with TBST, the membranes were developed using an enhanced chemiluminescence western blot detection system (Merck KGaA). Quantity One 4.62 (Bio-Rad Laboratories, Inc., Hercules, CA, USA) and Image J software (version 2.1; National Institutes of Health, Bethesda, MD, USA) was used analyze the results and calculate the expression of ARPC4 and $\beta$-actin. $\beta$-actin was used as a reference protein.

ARPC4-siRNA transfection. Cells were seeded in 6-well plates $\left(3.5 \times 10^{5}\right.$ cells per well). A negative control (NC) 
Table I. Oligonucleotide sequences of the siRNAs against actin-related protein 2/3 complex subunit 4 .

\begin{tabular}{llc}
\hline siRNA name & Sense & Antisense \\
\hline Negative control & UUCUCCGAACGUGUCACGUTT & ACGUGACACGUUCGGAGAATT \\
siRNA 496 & GAGCAGAGAACUUCUUUAUTT & UAAAGAAGUUCUCUGCUCTT \\
siRNA 538 & GGUAUGAUAUCAGCUUUCUTT & AGAAAGCUGAUAUCAUACCTT \\
siRNA 687 & GCUGAAGAGUUCCUUAAGATT & UCUUAAGGAACUCUUCAGCTT
\end{tabular}

siRNA, short interfering RNA.

group was transfected with non-silencing siRNA of the same length as ARPC4-siRNA. A null group was incubated under normal conditions (DMEM containing 10\% FBS) without siRNA transfection. Transfection was conducted using Lipofectamine ${ }^{\circledR} 2000$ according to the manufacturer's protocol. The GenBank database (https://www.ncbi.nlm. nih.gov/genbank/) was used to select the RNA interference target area for the ARPC4 gene, which was used for the design and synthesis of the three siRNA sequences and the NC (Shanghai GenePharma Co., Ltd., Shanghai, China). The siRNAs were designated as siRNA496, siRNA538 and siRNA679, according to the corresponding target sequence cDNA-initiation site, and BLAST (https://blast.ncbi.nlm. nih.gov/) queries were conducted to rule out homology with other genes (Table I).

Cell viability assays. Cells in the logarithmic growth phase immediately following transfection were seeded in 96-well plates $\left(1 \times 10^{3}\right.$ cells per well), with three wells allocated to each experimental group. The absorbance in each well was measured at 0, 1, 2, 3 and 4 days, using Cell Counting kit-8 (EnoGene Biotech Co., Ltd., Nanjing, China) according to the manufacturer's protocol. Absorbance was measured at $450 \mathrm{~nm}$ using a plate reader, and growth curves were generated from the resulting data.

Flow cytometry analysis of cell cycle distribution. A total of $48 \mathrm{~h}$ following transfection, cells from each experimental group were fixed overnight at $4^{\circ} \mathrm{C}$ with cold $70 \%$ ethanol prior to being incubated with RNase $\mathrm{A}$ at $37^{\circ} \mathrm{C}$ for $30 \mathrm{~min}$. The cells were stained with propidium iodide for $30 \mathrm{~min}$ in the dark at room temperature, and cell cycle analysis was conducted using a FACSCanto II flow cytometer (BD Biosciences, Franklin Lakes, NJ, USA) and ModFit LT software 4.1 (Verity Software House, Inc., Topsham, ME, USA).

Cell migration assays. Cell migration assays were performed using transwell chamber dishes $(6.5 \mathrm{~mm})$ with $8.0-\mu \mathrm{m}$ pore polycarbonate membrane inserts (EDM Millipore, Billerica, MA, USA). Serum-free cell suspensions (200 $\mu 1,10^{5}$ cells) were seeded in the upper compartment, while $450 \mu$ l DMEM containing $30 \%$ FBS was placed in the lower chamber. The cells were incubated for $48 \mathrm{~h}$. The upper membrane surface was wiped to remove cells, and the migrated cells on the lower surface were fixed with $4 \%$ methanol at room temperature for $30 \mathrm{~min}$ and stained with $0.1 \%(\mathrm{~g} / \mathrm{ml})$ crystal violet for $30 \mathrm{~min}$ at room temperature. Using a light microscope, cells in three random fields of vision (magnification, $\mathrm{x} 400$; DP-50; Olympus Corporation, Tokyo, Japan) were counted for each sample and means were reported.

Statistical analysis. Statistical analyses were performed using GraphPad Prism 5.0 (GraphPad Software, Inc., La Jolla, CA, USA) and SPSS 16.0 statistical software (SPSS, Inc., Chicago, IL, USA). All data are presented as the mean \pm standard deviation from 3 independent experiments. Student's t-test was used when comparing between two groups and one-way analysis of variance with Tukey's post-hoc test was used when comparing more than two groups. $\mathrm{P}<0.05$ was considered to indicate a statistically significant difference.

\section{Results}

Immunohistochemical analysis of ARPC4 expression in human carcinoma-associated antigen-containing tissues and adjacent non-tumour colorectal tissues. ARPC4 expression in colorectal carcinoma and normal colon tissues was assessed by immunohistochemistry. High levels of ARPC4 staining were observed in tumour tissues, whereas the normal tissues were negative for ARPC4 (Fig. 1).

Analysis of ARPC4 expression in colorectal cancer cells by western blot. Western blot results revealed that ARPC4 was expressed at high levels in SW620 and HT-29 cell lines relative to other cell lines (Fig. 2), with the highest expression observed in SW620 cells (Fig. 2). For this reason, the SW620 cell line was selected for subsequent experiments.

ARPC4-siRNA538 exhibited the strongest silencing effect. Western blot analysis was carried out to determine which of the ARPC4-siRNAs yielded the most effective silencing. As presented in (Fig. 3), siRNA538 resulted in the highest level of ARPC4 inhibition. and was, therefore, used in subsequent experiments. The inhibition rate was significantly decreased at all time points, but the optimal transfection time was $48 \mathrm{~h}$. (Fig. 4).

Effect of ARPC4 on viability. As determined from cell growth curves, cell viability rates did not differ significantly among the siRNA, NC, Lipo and Null groups (Fig. 5). Cell cycle analysis by flow cytometry yielded the following results for the siRNA group vs. the NC group: $\mathrm{G}_{0} / \mathrm{G}_{1}$ phase, 66.92 vs. $66.80 \%$; $S$ phase, 20.71 vs. $22.18 \%$; and $\mathrm{G}_{2} / \mathrm{M}$ phase, 12.37 vs. $11.03 \%$ (Fig. 6). Differences in the $G_{0} / G_{1}, S$, and $G_{2} / M$ 

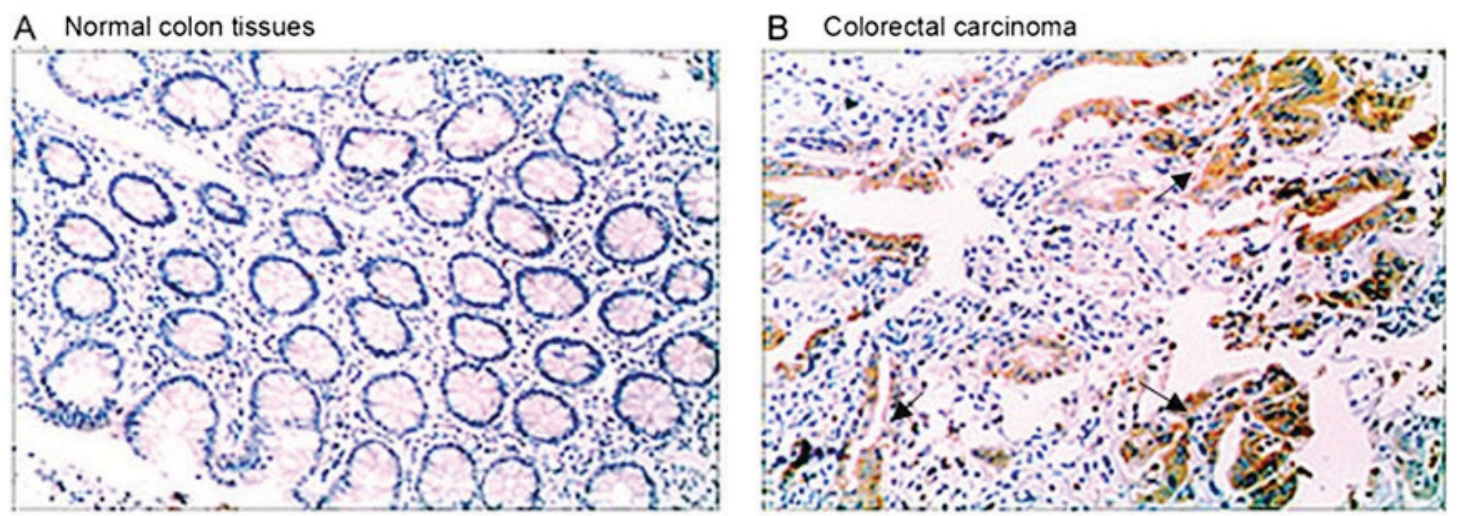

Figure 1. Immunohistochemical analysis of ARPC4 expression in (A) a colorectal carcinoma and (B) an adjacent normal colon tissue sample. Relatively high ARPC4 staining was observed in the tumour tissue, whereas the normal tissue was negative for ARPC4. ARPC4, actin-related protein $2 / 3$ complex subunit 4 .
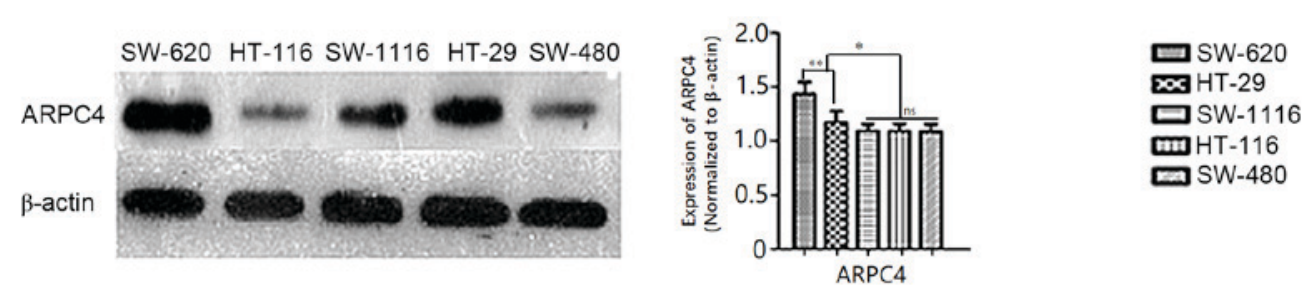

Figure 2. Representative image of relative ARPC4 expression levels in colorectal cancer cell lines were investigated using western blot analysis. ARPC4, actin-related protein 2/3 complex subunit 4. SW620 and HT-29 cell line groups demonstrated a relative higher ARPC4 expression levels when compare with the other groups ("P $<0.05$ vs. HT-116, SW1116, SW480 groups). However, the ARPC4 expression in SW620 group is much more apparent compared with the HT-29 group. (" $\mathrm{P}<0.01$ vs. HT-29 group). The differences were not significant among HT-116, SW1116, SW480 groups. " $\mathrm{P}<0.05$; ${ }^{* *} \mathrm{P}<0.01$; ns, not significant.
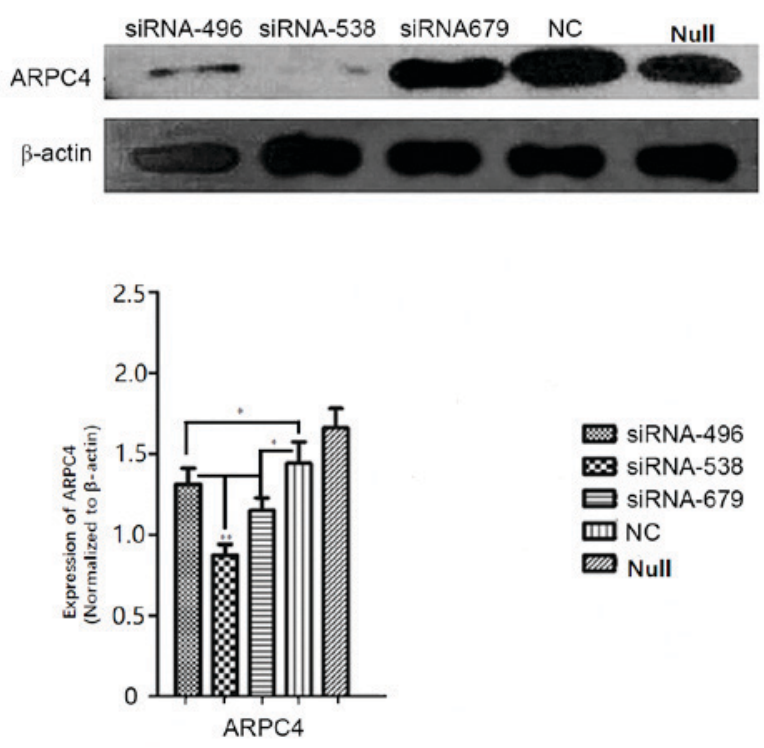

Figure 3. Comparison of silencing efficiencies for three ARPC4-siRNA oligonucleotide sequences. Comparison of ARPC4 protein expression levels among siRNA, NC and Null groups in SW620 cells after transfection with three ARPC4-siRNA oligonucleotide sequences and NC. Following transfection with siRNA and NC, silencing efficiencies between siRNA and NC groups were significant ("P<0.05 vs. NC). siRNA538 resulted in the highest level of ARPC4 inhibition when compared with the other siRNA groups $\left({ }^{* *} \mathrm{P}<0.01\right.$ vs. siRNA496/siRNA679). ARPC4, actin-related protein $2 / 3$ complex subunit 4; siRNA, short interfering RNA; NC, nonspecific siRNA; siRNA496, short interfering RNA with oligonucleotide sequences number 496; siRNA538, short interfering RNA with oligonucleotide sequences number 538; siRNA679, short interfering RNA with oligonucleotide sequences number 679; NC, nonspecific siRNA; null, control group; ${ }^{*} \mathrm{P}<0.05 ;{ }^{* *} \mathrm{P}<0.01$. phase distributions between the groups were not statistically significant. Furthermore, PCNA expression, as determined by western blot, did not differ significantly between groups (Fig. 7).

ARPC4 promotes $S W 620$ cell migration. To examine the effect of ARPC4 on SW620 cell migration, Transwell and western blot analyses were conducted. In the transwell assay, the number of migrated cells in siRNA group were significantly decreased compared with that observed in the NC group (transmembrane cell count, siRNA vs. NC: $20.4 \pm 1.14$ vs. $60.6 \pm 2.07$; $\mathrm{P}<0.05$; Fig. 8 ), while the differences among $\mathrm{NC} / \mathrm{Null} /$ Lipo groups were not significant (transmembrane cell count $60.6 \pm 2.07,50.0 \pm 1.58$ and $50.8 \pm 1.59 ; \mathrm{P}>0.05$ ). Western blotting results revealed that E-cadherin (Fig. 9A) and vimentin (Fig. 9B) expression differed significantly between the siRNA-transfected cells and the Null SW620 cells, respectively. It was therefore concluded that cells with ARPC4 knockdown exhibited a decreased metastatic capacity compared to Null/NC cells. NC, nonspecific siRNA; Null, control group; siRNA, small interfering target RNA; ns; not significant.

\section{Discussion}

While high levels of ARPC4 expression have been identified in colorectal cancer tissues, the association between the ARPC4 gene and the occurrence and development of colorectal cancer has not yet been elucidated (5). 

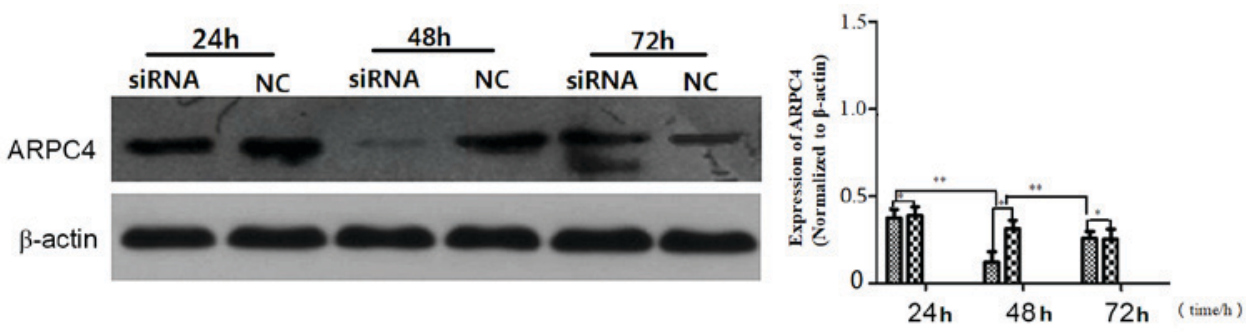

Figure 4. ARPC4 protein expression in SW620 cells following transfection with ARPC4-siRNA538. Comparison of ARPC4 protein expression silencing efficiencies at time point 24,48 and $72 \mathrm{~h}$ after transfected with siRNA538 and NC. The groups include: $24 \mathrm{~h}$ group (siRNA538/NC); $48 \mathrm{~h}$ group (siRNA538/NC); $72 \mathrm{~h}$ group (siRNA538/NC). The protein expression differences between siRNA538 and NC were statistically significant at each time point ("P $<0.05$ vs. NC). The siRNA538 silencing efficiencies at $48 \mathrm{~h}$ group were much more significant than at the other time points, the differences were statistically significant ( ${ }^{* *} \mathrm{P}<0.01$ vs. 24/72 h group). siRNA538, small interfering target RNA with oligonucleotide sequences number 538; NC, nonspecific siRNA; ${ }^{*} \mathrm{P}<0.05 ;{ }^{* *} \mathrm{P}<0.01$.
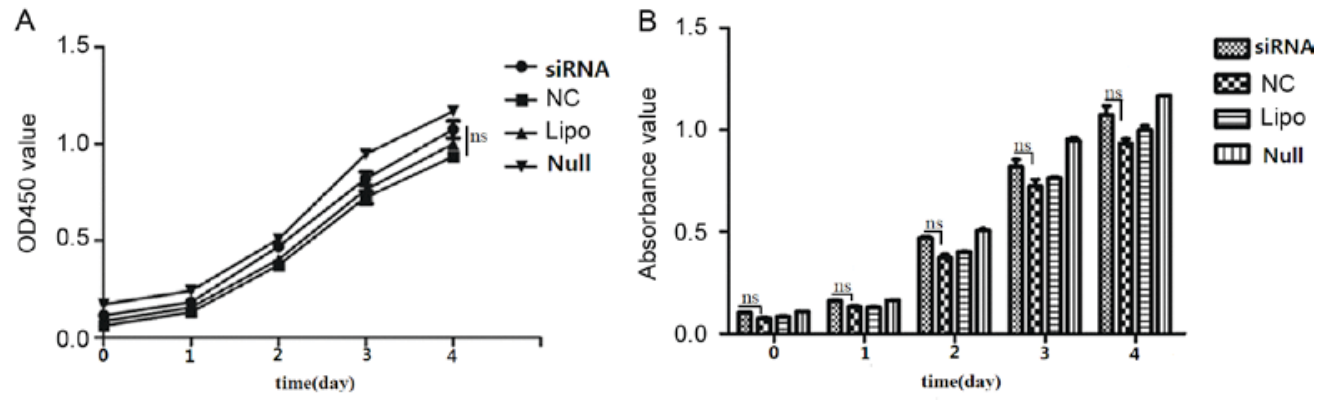

Figure 5. Growth curves for cells subsequent to actin-related protein 2/3 complex subunit 4 siRNA transfection. (A) OD ${ }_{450}$ value of each group. (B) Mean absorbance of each group. The relative proliferation rates at $0,1,2,3$ and 4 days subsequent to transfection in SW620 cells show no significant differences between the siRNA group and NC group (ns vs. NC). OD, optical density; siRNA, small interfering target RNA; NC, nonspecific siRNA; Lipo, Lipofectamine-only group; Null, control group; ns, not significant.

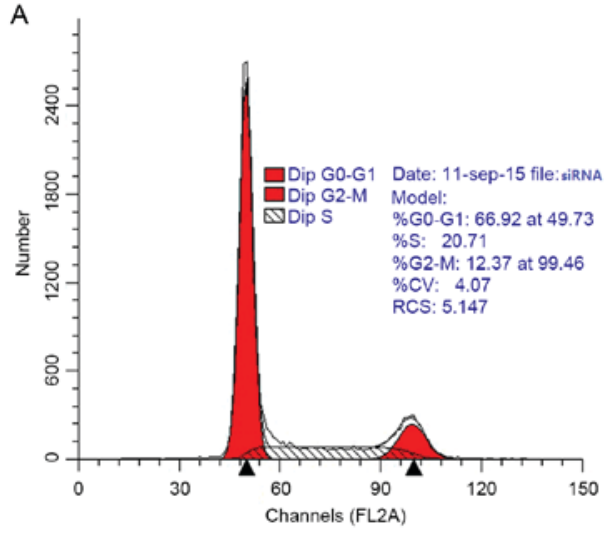

C

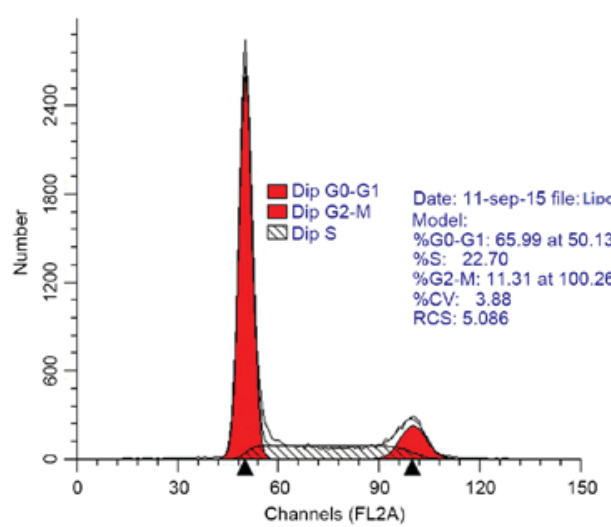

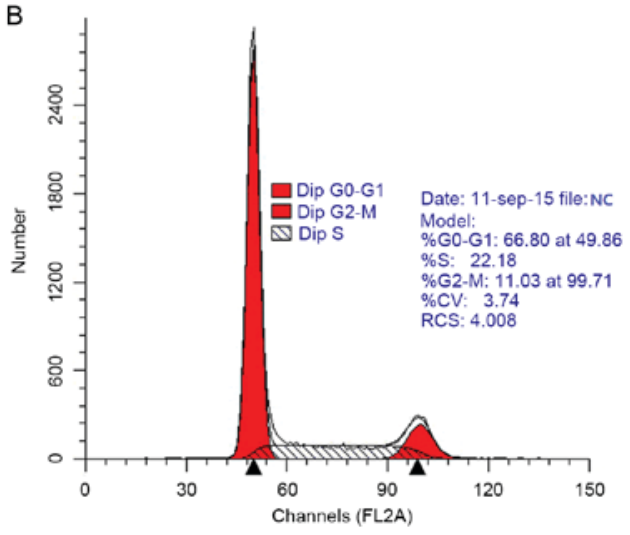

D

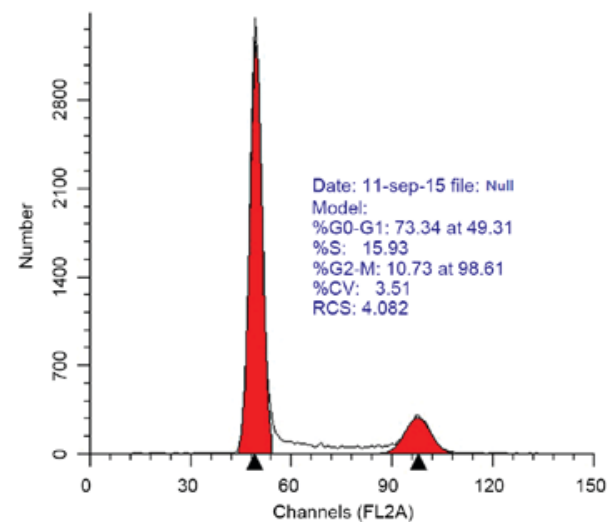

Figure 6. Cell cycle distribution analysis by flow cytometry. (A) siRNA group; (B) NC group; (C) Lipo group and (D) Null group $24 \mathrm{~h}$ after transfection of SW620 cells. Differences in the G0/G1, S and G2/M phase distribution were not statistically significant between the siRNA group and NC group (ns vs. NC). siRNA, small interfering target RNA; Null, control group; NC, nonspecific siRNA; Lipo, Lipofectamine-only group; ns, not significant. 

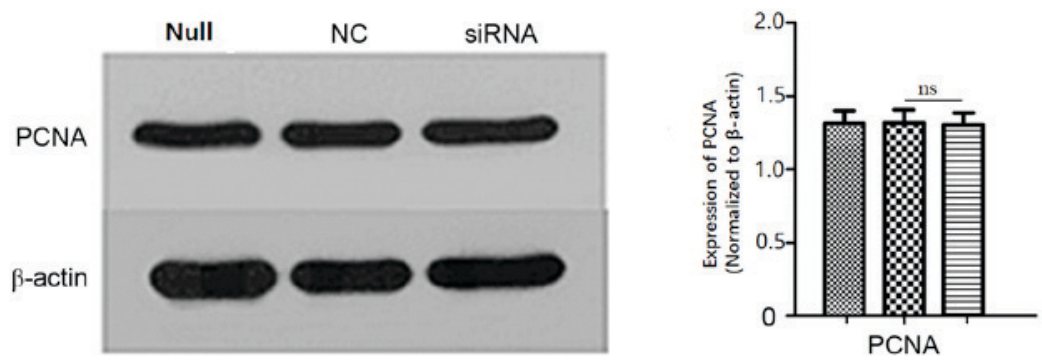

\section{Nul 吕 SiRNA}

Figure 7. Analysis of PCNA expression by western blotting subsequent to transfection with actin-related protein $2 / 3$ complex subunit 4 siRNA. PCNA expression differences in siRNA and NC groups of SW620 cells after transfected $48 \mathrm{~h}$ were not statistically significant (ns vs. NC). PCNA, proliferating cell nuclear antigen; siRNA, small interfering target RNA; Null, control group; NC, nonspecific siRNA; ns, not significant.

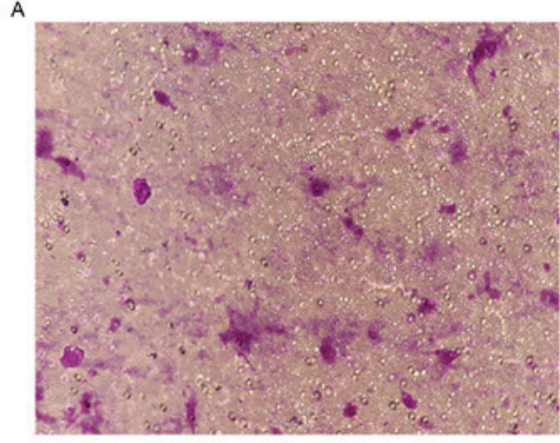

SIRNA

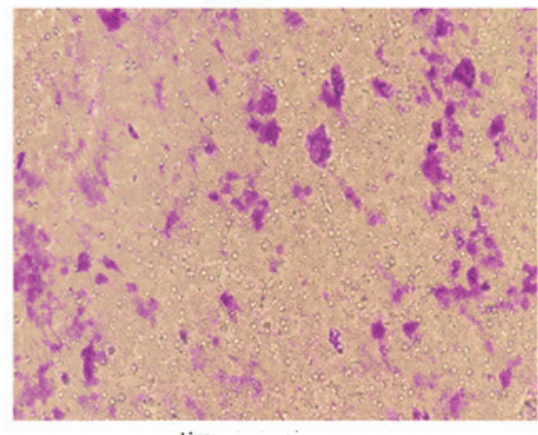

Lipo

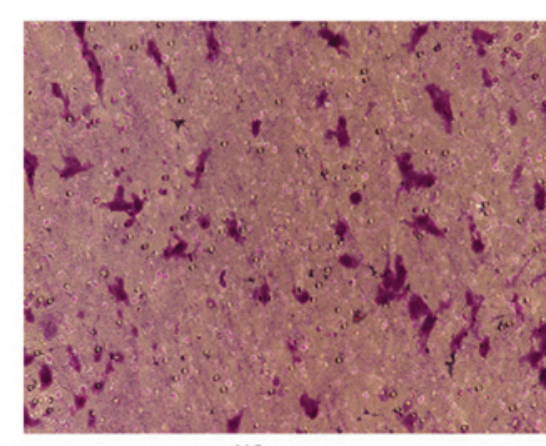

NC

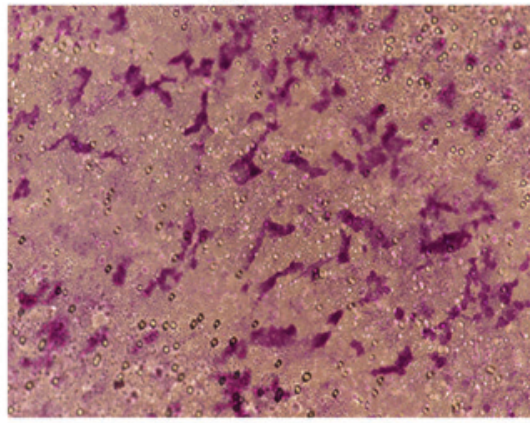

Null

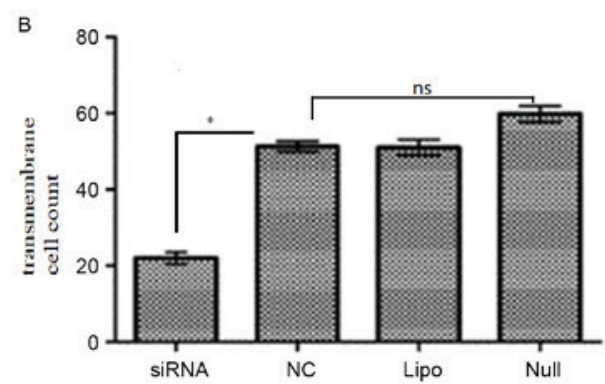

Figure 8. Transwell assay analysis of cell migration. (A) Representative images (magnification, x400) and (B) quantification of the Transwell cell migration assay. Comparing the transmembrane cell count in siRNA, NC, Lipo and Null groups of SW620 cells after transfected 48 h. The number of migrated cells in siRNA group were significantly decreased compared with that observed in the NC group (*P<0.05 vs. NC), while the differences were not statistically significant among Null group, Lipo group and NC group (ns vs. Null/Lipo). ${ }^{*} \mathrm{P}<0.05$. NC, negative control; Lipo, Lipofectamine-only group; siRNA, short interfering RNA group; ns, not significant.

Therefore, the present study explored the underlying molecular mechanisms of the function of ARPC4 in the progression of colorectal cancer and revealed that ARPC4 may serve a crucial function in colorectal cancer cell migration.

The findings of the present study indicated that although ARPC4-siRNA538 transfection did not influence cell viability, the invasiveness of cells transfected with siRNA538 was significantly diminished. The actin cytoskeleton formed by monomeric globular actin serves an essential function in several cellular processes, including division, migration, adhesion, and endocytosis. A number of these functions involve contact with the plasma membrane to allow the actin network outside of the cell to respond to extracellular signals. The aforementioned processes result from actin cytoskeleton 
A

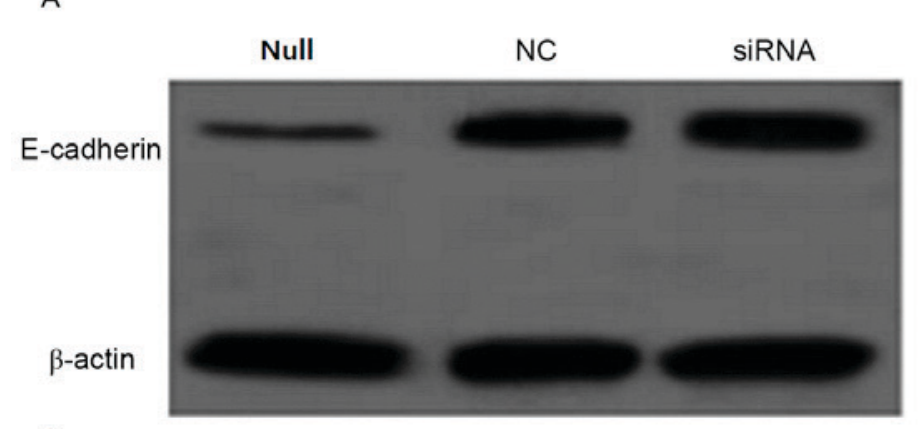

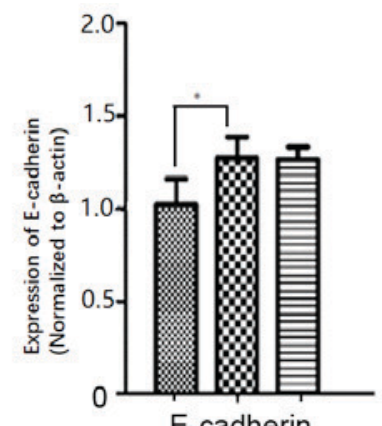

E-cadherin

B
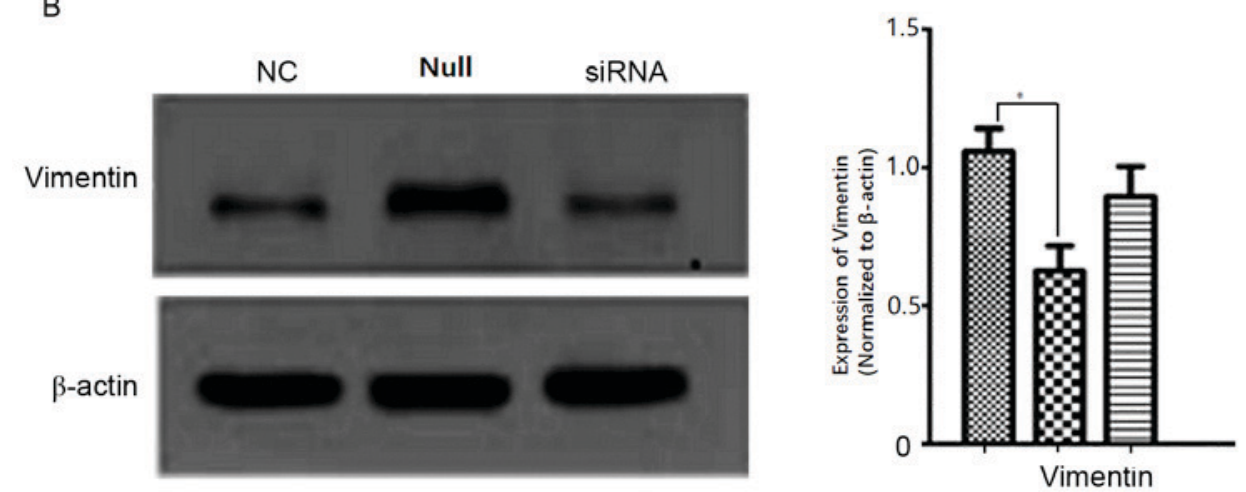

Figure 9. Analysis of E-cadherin and vimentin expression by western blot. (A) E-cadherin protein expression in Null, NC and siRNA groups of SW620 cells after transfected $48 \mathrm{~h}$. the protein expression show significant differences between siRNA group and Null group ("P $<0.05$ vs. Null), while the differences were not statistically significant between Null group and NC group. (B) vimentin protein expression in NC, Null and siRNA groups of SW620 cells after transfected $48 \mathrm{~h}$. The protein expression level show significant differences between siRNA group and Null group ("P<0.05 vs. Null), while the differences were not statistically significant between Null group and NC group. NC, nonspecific siRNA; Null, control group; siRNA, small interfering target RNA.

rearrangement, which involves numerous regulatory factors, including the ARP $2 / 3$ complex, which is an evolutionary conserved 220-kDa complex comprised of ARP2, ARP3, and five affiliated proteins (ARPC1-5) (6-10).

The ARP $2 / 3$ complex is an important component of the cytoskeleton that promotes the nucleation of new microfilaments and functions in the maintenance of cell shape, motility, and cytokinesis. ARPC4 and ARPC2 constitute the centre of the complex, whereas ARPC4 was previously demonstrated to serve an important function in the biological function of ARP2/3 in pancreatic cancer (11-14). ARPC4, the expression of which is abnormally high in colorectal cancer cell lines, regulates the actin nucleation process in cells, forms fusion proteins with the products of the downstream genes and influences the migration of pancreatic cancer cells $(15,16)$.

PCNA is a $36 \mathrm{kDa}$ protein that is only identified in the nuclei of normal proliferative and tumour cells. PCNA is associated with cell DNA synthesis and serves an important function in the initiation of cell proliferation (17). Tumour cells exhibit strong proliferative activity; PCNA may be used as an evaluation index of the cell proliferation state. In order to further determine the influence of ARPC4 on SW620 colorectal cancer cell proliferation in the present study, the PCNA protein expression level was investigated; its expression was not significantly different between groups.

The expression of E-cadherin was markedly increased, whereas the expression of vimentin was decreased in ARPC4-silenced cells compared with the control cells. E-cadherin is considered to be a tumour invasion and metastasis suppressor gene, and belongs to the calcium-dependent cadherin family. The expression of E-cadherin, which maintains the stability of the connection between normal cells, is negatively correlated with the occurrence of the epithelial-mesenchymal transition (EMT) and tumourigenesis. E-cadherin is connected to the cytoskeleton by its interaction with catenin to inhibit the proliferation of tumour cells and the production of matrix metalloproteinases by the host cell (18-20).

Additionally, E-cadherin prevents the degradation of various proteins of the matrix and basement membrane surrounding the tumour cells, thereby inhibiting tumour cell degradation of the matrix and basement membrane barriers (21-23). Invasion of tumour cells is regulated by tumour-matrix interactions. Expression of the ARP2/3 complex is associated with stromal cells in colorectal cancer, and therefore ARP $2 / 3$ expression enhances the motility between stromal cells and tumour cells, thereby providing a more suitable environment for invasion by these two cell types (24). Vimentin, however, is considered an interstitial cell marker, the expression of which correlates positively with the occurrence of EMT and with tumour oncogenesis. Vimentin is the dominant central fibre in mesenchymal cells and participates in the maintenance of cell integrity. Decreased E-cadherin expression is associated with elevated vimentin expression, and waveform protein expression may interfere with cell adhesion mediated by E-cadherin (25-27). Therefore, the results of the present study suggested that ARPC4 may enhance the expression of vimentin, whereas it may inhibit the expression of E-cadherin, and that the expression of ARPC4 may therefore have decreased cell adhesion to promote migration in tumour cells, thus serving a function in tumour development. 
In summary, the use of RNA interference may effectively suppress human ARPC4 expression in colorectal cancer SW620 cells, thereby inhibiting cell migration. These results suggested that specific targeting of ARPC4 may represent a potential treatment for colorectal cancer. Although previous studies demonstrated that ARPC4 influences the migration of pancreatic and colorectal cancer cells $(15,16)$, further experiments should be undertaken to define the function of ARPC4 during the initiation of colorectal cancer. Future research will include determination of the mechanism by which ARPC4 expression influences the biological behaviour of tumour cells. This information will enable the elucidation of novel targets for the treatment of colorectal cancer.

\section{Acknowledgements}

The present study was supported by the Scientific Project of Sichuan Province (grant no. 16ZC1671) and the Program Science and Technology Bureau of Chengdu China (grant no. 2015-HM01-00141-SF).

\section{References}

1. Ferlay J, Shin HR, Bray F, Forman D, Mathers C and Parkin DM: Estimates of worldwide burden of cancer in 2008: GLOBOCAN 2008. Int J Cancer 127: 2839-2917, 2010.

2. Inra JA and Syngal S: Colorectal cancer in young adults. Dig Dis Sci 60: 722-733, 2015 .

3. Gross K and Brand MI: Genetic predisposition to colorectal cancer. In: Common Surgical Diseases. New York, Springer, pp189-191, 2014

4. August DA, Ottow RT and Sugarbaker PH: Clinical perspective of human colorectal cancer metastasis. Cancer Metastasis Rev 3: 303-324, 1984

5. Belvitch P, Brown ME, Brinley BN, Letsiou E, Rizzo AN, Garcia JGN and Dudek SM: The ARP 2/3 complex mediates endothelial barrier function and recovery. Pulm Circ 7: 200-210, 2017.

6. Yamaguchi $\mathrm{H}$ and Condeelis $\mathrm{J}$ : Regulation of the actin cytoskeleton in cancer cell migration and invasion. Biochim Biophys Acta 1773: 642-652, 2007.

7. Le Clainche $\mathrm{C}$ and Carlier MF: Regulation of actin assembly associated with protrusion and adhesion in cell migration Physiol Rev 88: 489-513, 2008.

8. Firat-Karalar EN and Welch MD: New mechanisms and functions of actin nucleation. Curr Opin Cell Biol 23: 4-13, 2011.

9. Goley ED and Welch MD: The ARP2/3 complex: An actin nucleator comes of age. Nat Rev Mol Cell Biol 7: 713-726, 2006.

10. Bailly M, Ichetovkin I, Grant W, Zebda N, Machesky LM, Segall JE and Condeelis J: The F-actin side binding activity of the Arp2/3 complex is essential for actin nucleation and lamellipod extension. CurrBiol 11: 620-625, 2001.

11. Rauhala HE, Teppoi S, Niemelä S and Kallioniemi A: Silencing of the ARP2/3 complex disturbs pancreatic cancer cell migration. Anticancer Res 33: 45-52, 2013.

12. Welch MD, DePace AH, Verma S, Iwamatsu A and Mitchison TJ: The human Arp2/3 complex is composed of evolutionarily conserved subunits and is localized to cellular regions of dynamic actin filament assembly. J Cell Biol 138: 375-384, 1997.
13. Robinson RC, Turbedsky K, Kaiser DA, Marchand JB, Higgs HN, Choe S and Pollard TD: Crystal structure of Arp2/3 complex. Science 294: 1679-1684, 2001.

14. Higgs HN and Pollard TD: Regulation of actin filament network formation through ARP2/3 complex: Activation by a diverse array of proteins. Ann Rev Biochem 70: 649-676, 2001.

15. Otsubo T, Iwaya K, Mukai Y, Mizokami Y, Serizawa H, Matsuoka T and Mukai K: Involvement of Arp2/3 complex in the process of colorectal carcinogenesis. Mod Pathol 17: 461-467, 2004.

16. Ghosh A, Tousif S, Bhattacharya D, Samuchiwal SK, Bhalla K, Tharad M, Kumar S, Prakash P, Kumar P, Das G and Ranganathan A: Expression of the ARPC4 subunit of human Arp2/3 severely affects Mycobacterium tuberculosis growth and suppresses immunogenic response in murine macrophages. PLoS One 8: e69949, 2013.

17. Xiong Y, Zhang $\mathrm{H}$ and Beach D: D type cyclins associate with multiple protein kinases and the DNA replication and repair factor PCNA. Cell 71: 505-514, 1992.

18. Goodlad RA: Quantification of epithelial cell proliferation, cell dynamics and cell kinetics in vivo. Wiley Interdiscip Rev Dev Biol 6, 2017

19. Xie Y, Li P, Gao Y, Gu L, Chen L, Fan Y, Zhang F and Zhang X: Reduced E-cadherin expression is correlated with poor prognosis in patients with bladder cancer: A systematic review and meta-analysis. Oncotarget 8: 62489-62499, 2017.

20. Cao H, Xu E, Liu H, Wan L and Lai M: Epithelial-mesenchymal transition in colorectal cancer metastasis: A system review. Pathol Res Pract 211: 557-69, 2015.

21. Berx G and van Roy F: Involvement of members of the cadherin superfamily in cancer. Cold Spring Harb Perspect Biol 1: a003129, 2009.

22. Abu Taha A and Schnittler HJ: Dynamics between actin and the VE-cadherin/catenin complex: Novel aspects of the ARP2/3 complex in regulation of endothelial junctions. Cell Adh Migr 8: 125-135, 2014.

23. Chen A, Beetham H, Black MA, Priya R, Telford BJ, Guest J, Wiggins GA, Godwin TD, Yap AS and Guilford PJ: E-cadherin loss alters cytoskeletal organization and adhesion in non-malignant breast cells but is insufficient to induce an epithelial-mesenchymal transition. BMC Cancer 14: 552, 2014.

24. Kovacs EM, Goodwin M, Ali RG, Paterson AD and Yap AS: Cadherin-directed actin assembly: E-cadherin physically associates with the Arp2/3 complex to direct actin assembly in nascent adhesive contacts. Curr Biol 12: 379-382, 2002.

25. Nijkamp MM, Span PN, Hoogsteen IJ, van der Kogel AJ, Kaanders $\mathrm{JH}$ and Bussink $\mathrm{J}$ : Expression of E-cadherin and vimentin correlates with metastasis formation in head and neck squamous cell carcinoma patients. Radiother Oncol 99: 344-348, 2011.

26. Canel M, Serrels A, Frame MC and Brunton VG: E-cadherin-integrin crosstalk in cancer invasion and metastasis. J Cell Sci 126: 393-401, 2013.

27. Kuefer R, Hofer MD, Gschwend JE, Pienta KJ, Sanda MG, Chinnaiyan AM, Rubin MA and Day ML: The role of an $80 \mathrm{kDa}$ fragment of E-cadherin in the metastatic progression of prostate cancer. Clin Cancer Res 9: 6447-6452, 2003.

This work is licensed under a Creative Commons Attribution-NonCommercial-NoDerivatives 4.0 International (CC BY-NC-ND 4.0) License. 